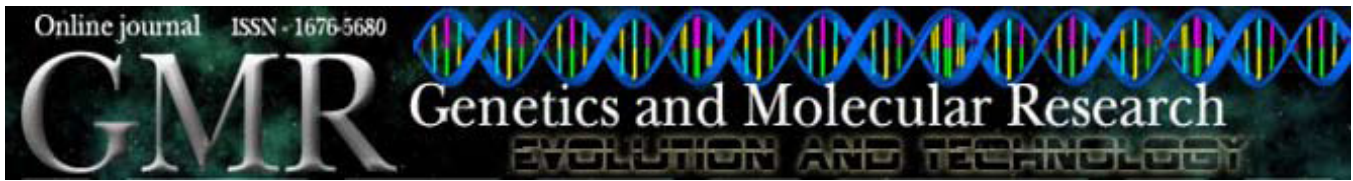

Thesis Abstract

\title{
Biochemical and molecular studies of copper metabolism during the infection process of the human pathogenic fungus Paracoccidioides brasiliensis
}

\author{
R.S. Santos \\ 2009. Pós-Graduação em Biologia Celular e Molecular, Instituto de Ciências \\ Biológicas, Universidade Federal de Goiás, Goiânia, GO, Brasil. Master's \\ thesis. Orienting Prof.: Dr. Célia Maria de Almeida Soares. Co-orienting Prof.: \\ Dr. Alexandre Melo Bailão.
}

The fungus Paracoccidioides brasiliensis is a human pathogen with a wide distribution in Latin America. The fungus causes paracoccidioidomycosis when mycelia reach the lungs. The success of the infection depends on the acquisition of essential micronutrients such as copper, which is required as a cofactor for a variety of enzymes important in several biological processes, such as respiration, growth and iron uptake. Previous studies of the Laboratory of Molecular Biology showed that a high-affinity copper transporter $(\mathrm{PbCTR} 3)$ is a molecule that is highly expressed and probably necessary for the establishment of infection by $P$. brasiliensis. In the present study, the genomic and cDNA sequences encoding PbCTR3 of $P$. brasiliensis were isolated and characterized. The cDNA consists of $582 \mathrm{bp}$ and encodes a protein of 193 amino acids, with a predicted molecular mass of $21.5 \mathrm{kDa}$ and $\mathrm{pI}$ of 8.6. The genomic sequence has four exons interrupted by three introns. In silico analysis was performed on the database of the structural genome of P. brasiliensis (http://www.broad.mit.edu/ annotation/genome/paracoccidioides_brasiliensisMultiHome.html), where genes involved in maintaining the homeostasis of copper have been identified and used to design a model of copper homeostasis in P. brasiliensis. The transcriptional behavior of Pbctr 3 and genes involved in copper homeostasis were examined during exposure of yeast cells of $P$. brasiliensis to conditions of copper and iron depletion, by real time qRT-PCR. A significant change was found in the transcription level of these genes in the absence of copper, as well as in the combined absence of both metals. qRT-PCR was used to analyze the expression of Pbctr3 and Pbcrp (which encodes a protein responsive to copper) in yeast cells of $P$. brasiliensis derived from infected lungs and spleen at different times of infection. Pbctr3 and Pbcrp were super-regulated during experimental infection. Taken together, these data suggest the importance of $P b C T R 3$ and of copper/iron uptake during the infection process.

Key words: Copper; Paracoccidioides brasiliensis; Gene expression and infection 